endorse the key role of family counseling and education of those who care for young children with febrile convulsions and they recommend that rectal diazepam be made available to the families of children with high recurrence risks. (McKinlay I, Newt on R. Intention to treat febrile convulsions with rectal diazepam, valproate or phenobarbitone. Dev Med Child Neurol October 1989; 31:617-625).

COMMENT. To treat or not to treat the febrile convulsion is a question that remains controversial. The results of this study are of interest but drug-level monitoring was performed infrequently; in children with recurrences the levels of valproate and phenobarbital were subtherapeutic in 40-60\% of cases.

In a more recent article concerning the use of long-term phenobarbital for febrile seizures (Farwell JR et al. N Engl J Med Feb 8, 1990; 322:364-9) the authors concluded that "phenobarbital depresses cognitive performance in children and that this disadvantage is not offset by the benefit of seizure prevention". The statistical design and analysis of this study is camplicated, requiring explanations for patient dropout rate and poor compliance. In those with seizure recurrence, blood phenobarbital levels were unavailable and parents had discontinued phenobarbital in one-third of cases.

Phenobarbital is the safest drug available and is prescribed for complex febrile seizures by $90 \%$ of pediatricians and family practitioners in Illinois. (Millichap JG et al. Ann Neurol (abstract) Sept $1989 ; 26: 473$ ). Significant reductions in seizure recurrence have been reported by several investigators when compliance is carefully controlled. Until new approaches to the management of febrile seizures can be defined by well controlled studies, the following recommendations are suggested: 1) Emphasis on parental counseling and education in the management of fever and convulsions. 2) Intermittent prophylactic therapy with oral diazepam, $0.5 \mathrm{mg} / \mathrm{kg} /$ daily in divided doses, at times of subsequent fever, as an alternative to long-term phenobarbital. 3) An approved rectal preparation of diazepam for the treatment of the acute febrile seizure in the home for use by selected parents in high risk patients. 4) Phenobarbital, when indicated in selected patients, should be limited to 6-12 months seizure free periods and monitored by blood levels ( $15 \mathrm{mcg} / \mathrm{ml}$ ) at six weeks to three month intervals and by behavioral and psychological evaluations before and during therapy.

\title{
RECURRENCE RISK AFTER A SINGLE SEIZURE
}

The risk of recurrence after a single, unprovoked, generalized tonic-clonic seizure was assessed in 119 children aged 2 to 16 years, resident in Normandy and exmined at the Hopital General, Le Havre and the Hopital Charles Nicolle, Rouen, France. All children in the study 
had a normal neurological examination and intellectual development, and the EEG showed no focal abnormality. The risk of recurrence at six months was $22 \%$, at one year $28.5 \%$, at three years $32.6 \%$ and at eight years $37.7 \%$. Fifty-eight percent of recurrences occurred within the first six months and $87 \%$ within the first two years. The risk of recurrence after two years in patients with EEGs showing spikes or spike-and-wave was $40 \%$ and this risk was not significantly different from that for 51 patients with normal EEGs (29\%). However, $73 \%$ of patients with abnormal EEGs were receiving treatment compared to $52 \%$ of those with normal EEGs. Phenobarbital was prescribed for 46 patients, sodium valproate 19 , carbamazepine 4 , phenytoin 1 , Trimedone 1 , and phenobarbital and clonazepam 2. Drug compliance was not evaluated. When the seizure lasted less than five minutes, the risk of recurrence at two years was $25 \%$ compared with $42 \%$ for those whose seizures lasted longer than five minutes. Age at onset of the initial seizure did not affect the risk of recurrence. Seizure duration and history of epilepsy in the family were not significant risk factors. In summary, single, short duration, convulsive seizures of the grand mal type should not be systematically treated, especially when clinical examination and EFG findings are normal. A diagnosis of epilepsy will be confirmed or disproved within two years of follow-up: if seizures do recur they usually do so within that period. A history of febrile seizures does not increase the risk of recurrence of a single unprovoked grand mal seizure. (Boulloche $J$ et al. Risk of recurrence after a single, unprovoked, generalized tonic-clonic seizure. Dev Med Child Neurol October 1989; $31: 626-663)$.

COMVENT. Seizures that are focal or associated with mental retardation or brain damage have a higher risk of recurrence and warrant prophylactic treatment with anticonvulsant drugs. A single unprovoked generalized tonic-clonic seizure does not require treatment but medication should be commenced if these seizures recur. In the present study, the cumulative risk of recurrence for treated patients was lower than that of untreated patients but the difference was not significant. One might speculate that the difference may have been significant if compliance with therapy had been monitored and adequate therapeutic drug levels maintained.

DRUG WITHDPAILAL AND RELAPSE RATE FOR GRAND MAL SEIZURES

The relapse rate when monotherapy was discontinued in 187 children who were seizure-free for three consecutive years was studied at the Leeds General Infirmary and St. James University Hospital, Leeds, England. Of a total group of 640 children with grand mal seizures $30 \%$ had became seizure-free on monotherapy. Children were assigned to one of three groups: 1) Normal neurologically and EEG; 2) normal neurologically, abnormal EFG; and 3) abnormal neurologically, including mental retardation with or wi thout abnormal EEG. Relapse occurred in 22 of the total $(12 \%)$. The relapse rate was the same in groups 1 and 2 and lower in the group 3 patients with abnormal 